\title{
Drug Accountability Numeric Result in Standard Unit
}

National Cancer Institute

\section{Source}

National Cancer Institute. Drug Accountability Numeric Result in Standard Unit. NCI Thesaurus. Code C87872.

The numerical identifier of a drug accountability assessment result in standard units. 